\title{
ON CONTINUOUS DEPENDENCE IN FINITE ELASTICITY*
}

\author{
BY SCOTT J. SPECTOR (University of Tennessee)
}

\begin{abstract}
We investigate the relation between stability and continuous dependence for a nonlinearly elastic body at equilibrium. We show that solutions of the governing equations that lie in a convex, stable set of deformations depend continuously on the body forces and the surface tractions. The definition of stability used is essentially due to Hadamard.
\end{abstract}

1. Introduction. In this note we consider the relation between stability and continuous dependence for a nonlinearly elastic body at equilibrium. We show that solutions of the governing equations that lie in a convex, uniformly Hadamard-stable set of deformations depend continuously on the body forces and surface tractions.

Gurtin and Spector [1] have shown that Hadamard stability is also sufficient for uniqueness of solutions of the governing equations. Thus uniqueness and continuous dependence follow from the same assumptions.

Hadamard stability of a set $\Omega$ of deformations is the requirement that for some $\alpha>0$

$$
\int_{\mathscr{B}} \nabla u \cdot A(\nabla f) \nabla u \geq \alpha\|\nabla u\|_{L^{2}(\mathscr{B})}^{2}
$$

for every deformation $f$ in $\Omega$ and every variation $u$. Here $A$ is the elasticity tensor, the derivative of the stress response function with respect to the deformation gradient, while $\mathscr{B}$ is the region of space occupied by the body in a fixed reference configuration.

2. The response function. Stability. We consider a body $\mathscr{B}$ and we identify $\mathscr{B}$ with the properly regular ${ }^{1}$ region of $\mathbb{R}^{3}$ it occupies in a fixed reference configuration. Further, we denote by $\mathscr{D}$ and $\mathscr{S}$ complementary subsets of the boundary $\partial \mathscr{B}$ with $\mathscr{D}$ non-empty and relatively open.

A deformation $f$ (of $\mathscr{B}$ ) will be a member of the space

$$
\operatorname{Def}=\left\{f \in C^{1}\left(\mathscr{B}, \mathbb{R}^{3}\right): \operatorname{det} \nabla f>0\right\},
$$

while a variation $u$ will be a smooth function $u: \mathscr{B} \rightarrow \mathbb{R}^{3}$ which satisfies

$$
u=0 \text { on } \mathscr{D} \text {. }
$$

Here det is the determinant and $\nabla$ the gradient operator in $\mathbb{R}^{3}$. In particular, $\nabla f$ is the tensor field with components $(\nabla f)_{i j}=\partial f_{i} / \partial x_{j}$.

\footnotetext{
* Received February 26, 1979. The author especially wishes to thank M. E. Gurtin for his comments on a previous version of this manuscript. Thanks are also due to $\mathbf{M}$. Aron and J. J. Roseman for correspondence concerning continuous dependence.

${ }^{1} \mathrm{Cf}$. Fichera [2], p. 351. In particular, $\mathscr{B}$ is compact and has piecewise smooth $\left(C^{1}\right)$ boundary.
} 
We assume that the body is elastic with smooth response function $S$. Thus

$$
S(\nabla f(x), x)
$$

gives the (Piola-Kirchhoff) stress at any point $x \in \mathscr{B}$ when the body is deformed by $f$. Writing $F$ for $\nabla f(x)$ we define the elasticity tensor $A(F, x)$ : Lin $\rightarrow$ Lin $^{2} y^{2}$

$$
A(F, x)=\partial_{F} S(F, x) \text {. }
$$

For convenience we write $S(\nabla f)$ and $A(\nabla f)$ for the fields on $\mathscr{B}$ with values $S(\nabla f(x), x)$ and $A(\nabla f(x), x)$ respectively.

Definition [1]. A set $\Omega \subset$ Def is (uniformly) Hadamard-stable or simply $H$-stable if for some $\alpha>0$

$$
\int_{\mathscr{B}} \nabla u \cdot A(\nabla f) \nabla u \geq \alpha\|\nabla u\|_{L^{2(B)}}^{2}
$$

for all $f \in \Omega$ and variations $u$, in which case we write $\operatorname{stab}(\Omega)$ for the largest $\alpha$ with this property.

In the above inequality

$$
\|\nabla u\|_{L^{2(B)}}^{2}=\int_{\mathscr{B}} \nabla u \cdot \nabla u
$$

where for any $T, V \in \mathrm{Lin}$,

$$
V \cdot T=\sum_{i, j} V_{i j} T_{i j} .
$$

We will also have occasion to use the $L^{2}$-norm of vector fields over $\mathscr{B}$ and $\mathscr{S}$;

$$
\|u\|_{L^{2}(\mathscr{B})}^{2}=\int_{\mathscr{B}} u \cdot u, \quad\|u\|_{L^{2}(\mathscr{S})}^{2}=\int_{\mathscr{S}} u \cdot u .
$$

Remark. The above notion of stability is essentially due to $\operatorname{Hadamard}^{3}$ [3, p. 252]. For a further discussion of stability cf. [1] and [4].

Gurtin and Spector [1] have shown that there is a neighborhood of the reference configuration which is uniformly $\mathrm{H}$-stable if either:

(a) the reference configuration is positive and natural ${ }^{4}$; or

(b) $\mathscr{D}=\partial \mathscr{B}$ and the reference configuration is homogeneous and strongly elliptic.

3. The mixed problem. Continuous dependence. The mixed problem (with dead loading) consists in finding a deformation $f$ that satisfies:

(i) the equation of virtual work

$$
\int_{\mathscr{B}} S(\nabla f) \cdot \nabla u=\int_{\mathscr{S}} s \cdot u+\int_{\mathscr{B}} b \cdot u
$$

for all variations $u$; and

${ }^{2}$ We use Lin to denote the space of linear transformations from $\mathbb{R}^{3}$ into $\mathbb{R}^{3}$.

${ }^{3}$ Hadamard stability is a static "criterion" for stability and its precise relation to dynamic stability is unclear. Eq. (2) cannot hold for all deformations, since global uniqueness would then follow.

${ }^{4}$ A natural configuration whose elasticity tensor is positive definite when restricted to symmetric tensors. 
(ii) the displacement boundary condition

$$
f=d \text { on } \mathscr{D} .
$$

Here, $d \in C^{0}\left(\mathscr{D}, \mathbb{R}^{3}\right)$ is the surface deformation, $s \in L^{2}\left(\mathscr{S}, \mathbb{R}^{3}\right)$ the surface traction, and $b \in L^{2}\left(\mathscr{B}, \mathbb{R}^{3}\right)$ the body force. The triplet $(d, s, b)$ will be referred to as the data, while a deformation $f \in$ Def that satisfies (3) and (4) will be called a solution corresponding to the data $(d, s, b)$.

Remark. The traction problem $(\mathscr{S}=\partial \mathscr{B}, \mathscr{D}=\varnothing)$ is excluded from our consideration since $\operatorname{stab}(\Omega)$ is, in general, zero when $\mathscr{D}$ is empty. For a partial resolution of this problem cf. [5].

Remark. If $s$ and $b$ are continuous and $f$ is a $C^{2}$ solution corresponding to the data $(d, s, b)$ then the divergence theorem can be used to show that (3) and (4) are equivalent to

$$
\begin{gathered}
\operatorname{div} S(\nabla f)+b=0 \text { in } \mathscr{B}, \\
f=d \text { on } \mathscr{D}, \quad S(\nabla f) n=s \text { on } \mathscr{S} .
\end{gathered}
$$

Note that the definition of stability is independent of the data although it does depend on $\mathscr{D}$, the domain of $d$.

Our main result is the following

THEOREM. Let $\Omega$ be convex ${ }^{5}$ and $H$-stable. Then there exists a constant $\lambda>0$, which depends only on $\operatorname{stab}(\Omega)$ and $\mathscr{B}$, such that if $f$ and $\hat{f}$ are solutions that lie in $\Omega$ and correspond to data $(d, s, b)$ and $(d, \hat{s}, \hat{b})$ respectively, then

$$
\|\hat{f}-f\|_{L^{2}(\mathscr{B})} \leq \lambda\left(\|\hat{s}-s\|_{L^{2}(\mathscr{S})}+\|\hat{b}-b\|_{L^{2}(\mathscr{B})}\right) .
$$

Remark. A direct consequence of our theorem is uniqueness: there is at most one solution corresponding to $(d, s, b)$ in $\Omega$. On the other hand, there may be additional solutions which do not lie in $\Omega$ and may therefore not depend continuously on the data. (For uniqueness under weaker hypotheses, cf. [1].)

Proof of the theorem. Let $\Omega \subset$ Def be convex and H-stable. Let $u=\hat{f}-f$. Then $\hat{f}=f$ on $\mathscr{D}$, so that $u=0$ on $\mathscr{D}$ and $u$ is a variation; hence (3) yields

$$
\int_{\mathscr{B}} \nabla u \cdot[S(\nabla \hat{f})-S(\nabla f)]=\int_{\mathscr{S}} u \cdot(\hat{s}-s)+\int_{\mathscr{B}} u \cdot(\hat{b}-b) .
$$

Consider the function $g:[0,1] \rightarrow \mathbb{R}$ defined by

$$
g(\sigma)=\int_{\mathscr{B}} \nabla u \cdot S(\nabla f+\sigma \nabla u)
$$

By (1)

$$
g^{\prime}(\sigma)=\int_{\mathscr{B}} \nabla u \cdot A(\nabla f+\sigma \nabla u) \nabla u .
$$

Hence, by the mean value theorem, $g(1)-g(0)=g^{\prime}(\xi)$ at some $\xi \in(0,1)$; so that

$$
\int_{\mathscr{B}} \nabla u \cdot[S(\nabla \hat{f})-S(\nabla f)]=\int_{\mathscr{B}} \nabla u \cdot A(\nabla f+\xi \nabla u) \nabla u .
$$

\footnotetext{
${ }^{5}$ Convexity here is with respect to the linear structure in $C^{1}\left(\mathscr{B}, \mathbb{P}^{3}\right)$.
} 
Since $\Omega$ is convex, $(f+\xi u) \in \Omega$ and therefore (2) gives us

$$
\operatorname{stab}(\Omega)\|\nabla u\|_{L^{2(B)}}^{2} \leq \int_{\mathscr{B}} \nabla u \cdot A(\nabla f+\xi \nabla u) \nabla u .
$$

Next, by the Cauchy-Schwarz inequality,

$$
\begin{aligned}
& \int_{\mathscr{B}} u \cdot(\hat{b}-b) \leq\|u\|_{L^{2}(\mathscr{B})}\|\hat{b}-b\|_{L^{2}(\mathscr{B})}, \\
& \int_{\mathscr{S}} u \cdot(\hat{s}-s) \leq\|u\|_{L^{2}(\mathscr{S})}\|\hat{s}-s\|_{L^{2}(\mathscr{S})} .
\end{aligned}
$$

Finally, the desired result follows from (5), (6), (7), (8) and the standard inequalities ${ }^{5}$

$$
\begin{aligned}
\|u\|_{L^{2}(\mathscr{B})} & \leq \lambda_{1}\|\nabla u\|_{\left.L^{2(\mathscr{B})}\right)}, \\
\|u\|_{L^{2(S)}}^{2} & \leq \lambda_{1}\left(\|u\|_{L^{2(\mathscr{B})}}^{2}+\|\nabla u\|_{\left.L^{2(\mathscr{B})}\right)}^{2},\right.
\end{aligned}
$$

where $\lambda_{1}>0$ depends only on $\mathscr{B}$.

Remark. It is clear from the proof of the theorem that the $L^{2}$-norm of $(\hat{f}-f)$ can be replaced by the $H^{1}$-norm:

$$
\|u\|_{H^{1(\mathscr{B})}}^{2}=\|u\|_{L^{2(\mathscr{B})}}^{2}+\|\nabla u\|_{L^{2(\mathscr{B})}}^{2} .
$$

In addition, if we replace (8) by ${ }^{7}$

$$
\begin{gathered}
\int_{\mathscr{B}} u \cdot(\hat{b}-b) \leq\|u\|_{H^{1}(\mathscr{D})}\|\hat{b}-b\|_{H^{-1}(\mathscr{B})}, \\
\int_{\mathscr{S}} u \cdot(\hat{s}-s) \leq\|u\|_{H^{1 / 2(\mathscr{S})}}\|\hat{s}-s\|_{H^{-1 / 2(\mathscr{S})}},
\end{gathered}
$$

and use the trace theorem (cf. e.g. [6, p. 277])

$$
\|u\|_{H 1 / 2(\mathscr{S})} \leq \lambda_{2}\|u\|_{H 1(\mathscr{B})},
$$

we arrive at the stronger result

$$
\|\hat{f}-f\|_{H^{1(\mathscr{B})}} \leq \lambda\left(\|\hat{s}-s\|_{H^{-1 / 2(\mathscr{S})}}+\|\hat{b}-b\|_{H^{-1(\mathscr{B})}}\right) .
$$

\section{REFERENCES}

[1] M. E. Gurtin and S. J. Spector, On stability and uniqueness in finite elasticity, Arch. Rat. Mech. Anal., to appear

[2] G. Fichera, Existence theorems in elasticity, in Handbuch der Physik, VIa/2, Berlin, Springer-Verlag (1972)

[3] J. Hadamard, Leçons sur la propagation des ondes et les equations d'hydrodynamique, Paris, Hermann (1903)

[4] S. J. Spector, On uniqueness in finite elasticity with general loading, J. Elasticity, to appear

[5] S. J. Spector, On uniqueness for the traction problem in finite elasticity, in preparation

[6] R. A. Adams, Sobolev spaces, New York, Academic Press (1975)

${ }^{6}$ The first is the Poincare inequality, cf., e.g., Fichera [2, p. 274, footnote 17], whose proof with minor modification applies in the present circumstance. The second is the trace theorem, cf., e.g., Fichera [2, p. 353], Adams [6, p. 113].

${ }^{7}$ The negative and fractional norms are defined in the usual manner. Cf., e.g., [6]. 